\title{
Low-Dose 3D Rotational Angiography in Measuring the Size of Intracranial Aneurysm: In Vitro Feasibility Study Using Aneurysm Phantom
}

Hee Jong Ki, $\mathrm{MD}^{1}$, Bum-soo Kim, MD, $\mathrm{PhD}^{2}$, Jun-Ki Kim, $\mathrm{MD}^{1}$, Jai Ho Choi, $\mathrm{MD}, \mathrm{PhD}^{1}$, Yong Sam Shin, $\mathrm{MD}, \mathrm{PhD}^{1}$, Yangsean Choi, $\mathrm{MD}^{2}$, Na-Young Shin, $\mathrm{MD}, \mathrm{PhD}^{2}$, Jinhee Jang, $\mathrm{MD}, \mathrm{PhD}^{2}$, Kook-jin Ahn, $\mathrm{MD}, \mathrm{PhD}^{2}$

${ }^{1}$ Department of Neurosurgery, The Catholic University of Korea, Seoul St. Mary's Hospital, Seoul, Korea

${ }^{2}$ Department of Radiology, The Catholic University of Korea, Seoul St. Mary's Hospital, Seoul, Korea

Purpose: Three-dimensional (3D) measurement of intracranial aneurysms is important in planning endovascular treatment, and 3D rotational angiography (RA) is effective in accurate measurement. The purpose of this study was to evaluate the feasibility of low dose 3D RA ( 5 seconds $0.10 \mu \mathrm{Gy} /$ frame) in measuring an intracranial aneurysm using an in vitro phantom.

Materials and Methods: We investigated an in vitro 3D phantom of an intracranial aneurysm with 10 acquisitions of 3D RA with a conventional dose ( 5 seconds $0.36 \mu G y / f r a m e)$ and 10 acquisitions with a low-dose (5 seconds $0.10 \mu \mathrm{Gy} /$ frame). 3D size and neck diameters of the aneurysm were measured and compared between the 2 groups (conventional and low-dose) using noninferiority statistics.

Results: The aneurysm measurements were well-correlated between the 2 readers, and noninferiority in the measurement of aneurysmal size of low-dose 3D RA was demonstrated, as the upper margin of the 1-sided $97.5 \%$ confidence interval did not cross the pre-defined noninferiority margin of $0.2 \mathrm{~mm}$ by the 2 readers.

Conclusion: Low-dose (5 seconds $0.10 \mu \mathrm{Gy} /$ frame) cerebral 3D RA is technically feasible and not inferior in in vitro 3D measurement of an intracranial aneurysm. Thus, low-dose 3D RA is promising and needs further evaluation for its clinical utility in the planning of endovascular treatment of an intracranial aneurysm.

Key Words: Cerebral angiography; Radiation dosage; Intracranial aneurysm

\section{INTRODUCTION}

Three-dimensional (3D) rotational angiography (RA) has a pivotal role in the evaluation of intracranial aneurysms, including accurate assessment of size as well as detection and pretreatment evaluation of aneurysms. ${ }^{1-6}$ Although there have been reports showing the feasi- bility of low-dose 3D RA, little work has been done to study the effectiveness of accurate measurement of an aneurysm using low-dose 3D RA. ${ }^{7,8}$

In this brief report, we aimed to evaluate the feasibility of the lowest possible dose of 3D RA (5 seconds $0.10 \mu \mathrm{Gy}$ / frame) by our machine to measure an intracranial aneurysm. We investigated

\section{Correspondence to:} Bum-soo Kim, MD, PhD Department of Radiology, The Catholic University of Korea, Seoul St. Mary's Hospital, 222 Banpo-daero, Seocho-gu, Seoul 06591, Korea

Tel: +82-2-2258-6239

Fax: +82-2-599-6771

E-mail: bkim.neurorad@gmail.com

Received: November 30, 2020

Revised: January 4, 2021

Accepted: February 4, 2021
Copyright $\odot 2021$ Korean Society of Interventional Neuroradiology This is an Open Access article distributed under the terms of the Creative Commons Attribution Non-Commercial License (http://creativecommons.org/licenses/by-nc/4.0) which permits unrestricted non-commercial use, distribution, and reproduction in any medium, provided the original work is properly cited. 
an in vitro phantom of an intracranial aneurysm, and the measurements were compared with those from 3D RA with a conventional dose ( 5 seconds $0.36 \mu \mathrm{Gy} /$ frame).

\section{MATERIALS AND METHODS}

The 3D replica of an intracranial aneurysm was designed by a vascular neurosurgeon (JHC) and printed with a 3D printer (Projet MJP 2500 Plus 3D; 3D Systems, Rock Hill, SC, USA) using an elastic polymer rubber material. It was originally printed for education and hands-on purposes for endovascular treatment of a large aneurysm with a complex vasculature, and the detailed description of making this 3D replica will be described separately. For the in vitro phantom study, a 3D replica was filled with iodixanol $270 \mathrm{mg} / \mathrm{mL}$ (Visipaque 270; GE Healthcare, Amersham, England) and placed in a saline chamber. Using a biplane flat detector angiography unit (Axiom Artis Zee; Siemens, Erlangen, Germany), a total of 20 acquisitions of 3D RA were performed; 10 acquisitions with a conventional dose ( 5 seconds $0.36 \mu \mathrm{Gy} /$ frame) and 10 acquisitions with a low-dose ( 5 seconds $0.10 \mu \mathrm{Gy} /$ frame). The common parameters were as follows: 5 -second rotation; rotation angle, 200 degrees with 1.5 -degree increments resulting in 133 projections; rotation speed, 40 degree/s; acquisition matrix, 1,240×960. Acquired data were sent to the workstation (Syngo 3D workplace; Siemens AG, Erlangen, Germany) and $3 \mathrm{D}$ reconstruction was performed with the following parameters; kernel type, Hounsfield unit; image characteristics, normal; reconstruction mode, native; slice matrix, 512×512, and voxel size, $0.47 \mathrm{~mm}$.

All the volume rendering (VR) images were displayed using factory-default settings for the visualization of an intracra- nial aneurysm, in an attempt for consistency of the datasets measured. Twenty sets of $V R$ reconstruction images were sorted in random order and investigated in blinded evaluation separately by 2 experienced neurointerventionists (BSK, HJK). Height, width, depth, and neck size of the aneurysm were measured on a dedicated workstation.

For the statistical analysis for the phantom study, noninferiority tests were used for the comparison of measured parameters of the aneurysm between group 1 with conventional 3D RA (0.36 $\mu \mathrm{Gy} /$ frame) and group 2 with low-dose 3D RA (0.10 $\mu \mathrm{Gy} /$ frame). Because there has been no general use of predefined noninferiority margin of size measurement of an aneurysm, we set $0.2 \mathrm{~mm}$ as an acceptable noninferiority margin. Interrater reliability was tested using the Cronbach a.

\section{RESULTS}

The measured values of $V R$ reconstructed images of the $3 D$ replica by 2 neurointerventionists are summarized in Table 1 . The mean size of the aneurysm neck ( \pm standard deviation) was $8.23 \pm 0.08 \mathrm{~mm}$ in group $1,8.20 \pm 0.07 \mathrm{~mm}$ in group 2 by reader 1 , and $8.11 \pm 0.04 \mathrm{~mm}, 8.06 \pm 0.05 \mathrm{~mm}$ by reader 2 , respectively. The mean height was $11.33 \pm 0.04 \mathrm{~mm}$ in group 1, $11.31 \pm 0.09 \mathrm{~mm}$ in group 2 by reader 1 , and $10.99 \pm 0.13 \mathrm{~mm}$, $10.94 \pm 0.08 \mathrm{~mm}$ by reader 2 , respectively. The mean width was $12.01 \pm 0.03 \mathrm{~mm}$ in group $1,11.92 \pm 0.08 \mathrm{~mm}$ in group 2 by reader 1 , and $11.94 \pm 0.07 \mathrm{~mm}, 11.90 \pm 0.05 \mathrm{~mm}$ by reader 2 , respectively. The mean depth was $14.42 \pm 0.06 \mathrm{~mm}$ in group 1 , $14.40 \pm 0.07 \mathrm{~mm}$ in group 2 by reader 1 , and $14.76 \pm 0.05 \mathrm{~mm}$, $14.68 \pm 0.04 \mathrm{~mm}$ by reader 2 , respectively.

The measured aneurysm sizes were well-correlated between the 2 readers (group 1, Cronbach's a=0.997; group 2,

\section{Table 1. Descriptive statistics of measured parameters of 3D replica according to readers}

\begin{tabular}{lccccc}
\hline \multirow{2}{*}{ Reader and group } & No. of observations & \multicolumn{3}{c}{ Diameters } \\
\cline { 3 - 5 } & & Neck & Height & Width \\
\hline Reader 1 & 10 & $8.23 \pm 0.08$ & $11.33 \pm 0.04$ & $12.01 \pm 0.03$ & $14.42 \pm 0.06$ \\
Group 1 & 10 & $8.20 \pm 0.07$ & $11.31 \pm 0.09$ & $11.92 \pm 0.08$ & $14.40 \pm 0.07$ \\
Group 2 & & & & $11.94 \pm 0.07$ & $14.76 \pm 0.05$ \\
Reader 2 & 10 & $8.11 \pm 0.04$ & $10.99 \pm 0.13$ & $11.90 \pm 0.05$ & $14.68 \pm 0.04$ \\
Group 1 & 10 & $8.06 \pm 0.05$ & $10.94 \pm 0.08$ & & \\
Group 2
\end{tabular}

Values are presented as mean \pm standard deviation.

3D, three-dimensional; AP, anteroposterior. 
Cronbach's $a=0.997)$. Moreover, the noninferiority in measurements (aneurysm neck, height, width, and depth) of lowdose 3D RA (0.10 $\mu \mathrm{Gy} /$ frame) was demonstrated, since the upper margin of the 1-sided $97.5 \%$ confidence interval did not cross the predefined noninferiority margin of $0.2 \mathrm{~mm}$ (Fig. 1).

\section{DISCUSSION}

In measuring the size of the aneurysm in our in vitro phantom study, the measured values in group 1 ( 5 seconds $0.36 \mu \mathrm{Gy} /$ frame; Fig. 1A) tended to be larger than those of group 2 ( 5 seconds $0.10 \mu \mathrm{Gy} /$ frame; Fig. 1B), but the margin of the 1-sided $97.5 \%$ confidence interval did not cross the predefined noninferiority margin of $0.2 \mathrm{~mm}$. Therefore, we showed that low-dose 3D RA was not inferior in terms of measuring aneurysm size. Pearl et al. ${ }^{8}$ evaluated 3D RA of varying acquisition time and dose in 3 swines and compared mean differences in arterial diameter measurements between protocols. Their results showed that measurement differences between the standard and low dose protocols were not clinically significant $(<0.5 \mathrm{~mm})$. Although it was the measurement of arterial diameter, their results were similar to our results with an intracranial aneurysm. For the measurement of an intracranial aneurysm, Pearl et al. ${ }^{7}$ also evaluated the difference in measurement between conventional and various low dose 3D-RA protocols. In their reports, there were differences of more than $0.5 \mathrm{~mm}$ between the 5 seconds 0.36 $\mu \mathrm{Gy} / \mathrm{frame}$ and the 5 seconds $0.10 \mu \mathrm{Gy} / \mathrm{frame}$. However, the comparison between these 2 protocols was just the range of difference from a single measurement of only 4 subjects and was not validated as a statistical comparison of measurements. On the contrary, this study had repeated acquisitions of 3D RA of an aneurysm phantom, and analysis was done with noninferiority statistics to show low-dose 3D RA using $0.10 \mu \mathrm{Gy} /$ frame was not inferior in terms of 3D measurement of aneurysm size by the margin of $0.2 \mathrm{~mm}$, which seemed to be clinically acceptable.

Theoretically, by changing the 3D RA protocol from group

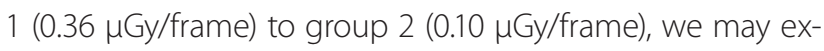
pect about a $72 \%$ of reduction of radiation dose from the acquisition of each 3D RA. In the report of Choi et al. ${ }^{9}$, the proportion of dose-area-product of 3D RA over total patient radiation dose ranged from $18 \%$ to $36 \%$. Therefore, by clinical application of a new protocol with low-dose 3D RA in the evaluation of an intracranial aneurysm, we may estimate a $13-26 \%$ reduction in total patient radiation dose. However, it should be validated in a patient cohort; furthermore, the evaluation of subjective image quality, including the visibility of small branches or perforating vessels, should be further evaluated by an in vivo study.

There are some limitations in this brief report. First, the aneurysm phantom was located in a saline chamber during the acquisition of 3D RA, thus attenuation of X-ray by the skull

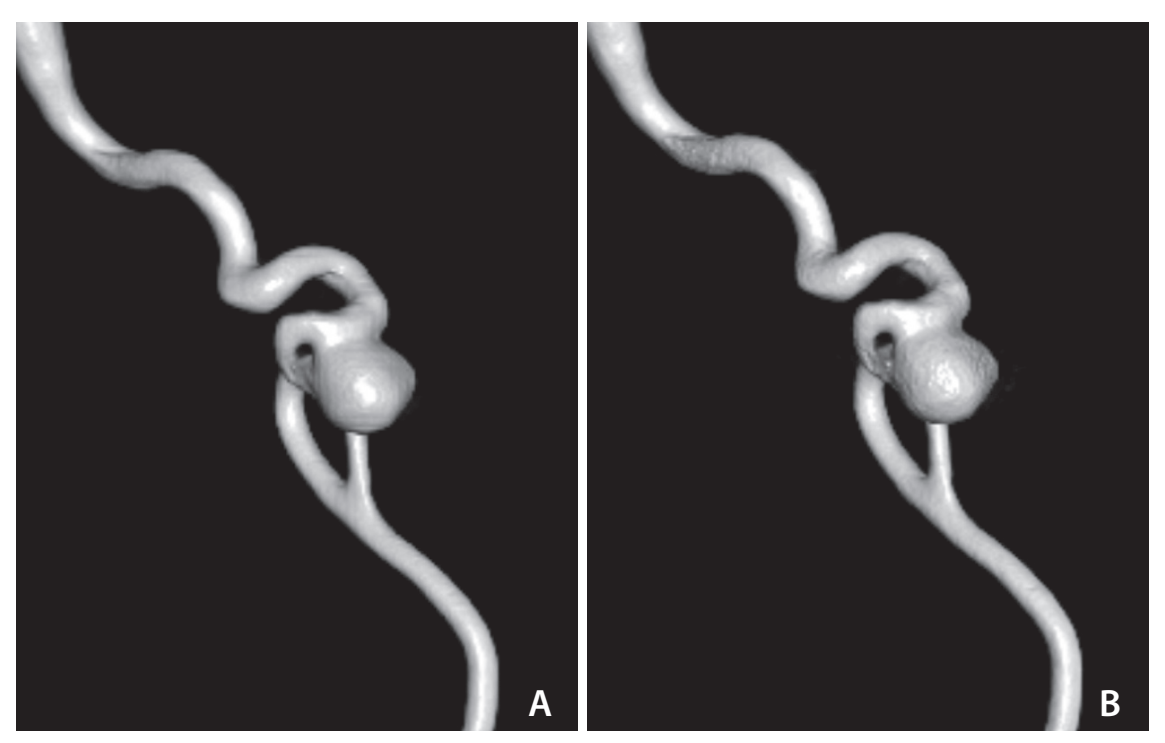

Fig. 1. Volume-rendering reconstruction images of in vitro $3 \mathrm{D}$ rotational angiography of intracranial aneurysm phantom. (A) Conventional dose with 5 seconds $0.36 \mu \mathrm{Gy} /$ frame (group 1); (B) low-dose with 5 seconds $0.10 \mu \mathrm{Gy} /$ frame (group 2). 3D, three-dimensional. 
could not be considered in this in vitro study. Second, pulsatile flow was not applied to the aneurysm phantom. Pulsatile movement of the vascular wall averaged during 5 seconds of 3D RA acquisition could create artifacts, which could be more prominent with low-dose radiation. But, it was evaluated in this study with a relatively rigid phantom without pulsatile flow. Third, although repeated measurements of 3D RA were performed, only a single replica of an intracranial aneurysm phantom was used. We assume that the in-vivo evaluation of a larger number of aneurysms in the patient cohort may overcome these limitations.

\section{CONCLUSION}

In conclusion, low-dose ( 5 seconds $0.10 \mu \mathrm{Gy} /$ frame) cerebral 3D RA was technically feasible and not inferior in the in vitro 3D measurement of an intracranial aneurysm, with a considerable reduction in radiation dose. Thus, low-dose 3D RA is promising and needs further evaluation for its clinical utility in the planning of endovascular treatment of an intracranial aneurysm.

\section{Fund}

None.

\section{Ethics Statement}

This study waived approval of the institutional ethics committee.

\section{Conflicts of Interest}

BK has been the Associate Editor of the Neurointervention since 2020. No potential conflict of interest relevant to this article was reported.

No other authors has any conflict of interest to disclose.

\section{Author Contributions}

Concept and design: HJK, BK, JK, JHC, YSS, JJ, and KA. Analysis and interpretation: HJK, BK, JHC, YSS, YC, and KA. Data collection: HJK, BK, JK, JHC, and YSS. Writing the article: HJK and BK. Critical revision of the article: HJK, BK, JHC, YSS, YC, NS, JJ, and KA. Final approval of the article: HJK, BK, JK, JHC, YSS, YC, NS, JJ, and KA. Statistical analysis: HJK, BK, YC, and NS. Overall responsibility: HJK and BK.

\section{ORCID}

Hee Jong Ki: https://orcid.org/0000-0003-0914-5516

Bum-soo Kim: https://orcid.org/0000-0002-3870-6813

Jun-Ki Kim: https://orcid.org/0000-0003-4822-4866

Jai Ho Choi: https://orcid.org/0000-0001-6838-0343

Yong Sam Shin: https://orcid.org/0000-0002-4250-1896

Yangsean Choi: https://orcid.org/0000-0003-1674-7101

Na-Young Shin: https://orcid.org/0000-0003-1157-6366

Jinhee Jang: https://orcid.org/0000-0002-3386-1208

Kook-Jin Ahn: https://orcid.org/0000-0001-6081-7360

\section{REFERENCES}

1. Gauvrit JY, Leclerc X, Vermandel M, Lubicz B, Despretz D, Lejeune JP, et al. 3D rotational angiography: use of propeller rotation for the evaluation of intracranial aneurysms. AJNR Am J Neuroradiol 2005;26:163-165

2. Hochmuth A, Spetzger U, Schumacher M. Comparison of three-dimensional rotational angiography with digital subtraction angiography in the assessment of ruptured cerebral aneurysms. AJNR Am J Neuroradio/ 2002;23:1199-1205

3. Abe T, Hirohata M, Tanaka N, Uchiyama Y, Kojima K, Fujimoto $K$, et al. Clinical benefits of rotational 3D angiography in endovascular treatment of ruptured cerebral aneurysm. AJNR Am J Neuroradiol 2002;23:686-688

4. Sugahara T, Korogi Y, Nakashima K, Hamatake S, Honda S, Takahashi M. Comparison of 2D and 3D digital subtraction angiography in evaluation of intracranial aneurysms. AJNR Am J Neuroradio/ 2002;23:1545-1552

5. van Rooij WJ, Sprengers ME, de Gast AN, Peluso JP, Sluzewski $M .3 D$ rotational angiography: the new gold standard in the detection of additional intracranial aneurysms. AJNR Am J Neuroradiol 2008;29:976-979

6. Anxionnat R, Bracard S, Ducrocq X, Trousset Y, Launay L, Kerrien E, et al. Intracranial aneurysms: clinical value of 3D digital subtraction angiography in the therapeutic decision and endovascular treatment. Radiology 2001;218:799-808

7. Pearl MS, Torok C, Katz Z, Messina SA, Blasco J, Tamargo RJ, et al. Diagnostic quality and accuracy of low dose 3D-DSA protocols in the evaluation of intracranial aneurysms. J Neurointerv Surg 2015;7:386-390

8. Pearl MS, Torok CM, Messina SA, Radvany M, Rao SN, Ehtiati T, et al. Reducing radiation dose while maintaining diagnostic image quality of cerebral three-dimensional digital subtraction angiography: an in vivo study in swine. J Neurointerv Surg 
Ki HJ et al. In Vitro Aneurysmal Phantom Study of Low Dose 3D RA

2014;6:672-676

9. Choi J, Kim B, Choi Y, Shin NY, Jang J, Choi HS, et al. Image quality of low-dose cerebral angiography and effectiveness of clinical implementation on diagnostic and neurointerventional procedures for intracranial aneurysms. AJNR Am J Neuroradiol 2019:40:827-833 